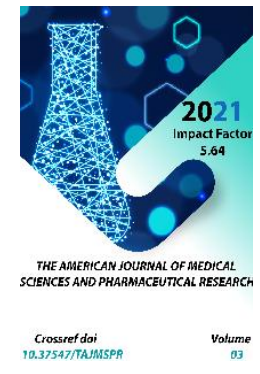

\title{
Torch-Infections As An Actual Problem In Obstetrics And Gynecology Practice
}

\author{
Tishabaeva Nargiza Alimdjanovna \\ Assistant, Department Of "Normal And Topographic Anatomy", Fergana Medical Institute of \\ Public Health, Ferghana, Uzbekistan
}

\section{ABSTRACT}

The article under discussion depicts TORCH infections as an actual problem in obstetrics and gynaecology practice. The author of the article considers that the course of the disease is often asymptomatic, and the pathological impact of the causative agents on the embryonic development of the fetus leads to the development of defects, which cause spontaneous abortions, perinatal and postnatal deaths, as well as a disability at a young age.

\section{KEYWORDS}

TORCH-infections, the pathological impact, causative, disease, spontaneous abortions, development of defects, postnatal deaths, mycoplasmosis, ureaplasmosis, chlamydia, DNA, RNA.

\section{INTRODUCTION}

TORCH-infections are a pressing problem in medicine since the causative agents of these diseases are widespread in the population, the course of the disease is often asymptomatic, and the pathological impact of the causative agents on the embryonic development of the fetus leads to the development of defects, which cause spontaneous abortions, perinatal 
and postnatal deaths, as well as a disability at a young age.

TORCH-infections are abbreviations of Latin names of intrauterine infections, i.e.:

\section{T - Toxoplasmosis (Toxoplasmosis)}

O - Other infections, that is, mycoplasmosis, ureaplasmosis, chlamydia, and others.

R - Rubella

C - Cytomegalia

$\mathrm{H}$ - Herpes (infections caused by herpes simplex virus).

The source of the infection process in this way can be a variety of pathogens: viruses, bacteria, parasitic and fungal infections.

In the vast majority of cases, the mother is the source of infection for the fetus. The mechanism of transmission is vertical. Transovarian, transplacental and ascending routes of transmission may occur during the antenatal period and contact, alimentary and intrapartum transmission may occur during the intrapartum period.

Transplacental (hematogenous) route infection passes from the mother to the fetus through the placenta. Most often transmitted viral intrauterine infections, as the virus easily penetrates the blood-placental barrier (like toxoplasma).

Ascending - an infection from the reproductive tract enters the uterine cavity and can then infect the fetus. Most often these are bacterial infections, sexually transmitted diseases, chlamydia, mycoplasmas. The downward route is from the fallopian tubes into the uterine cavity. The contact (intrapartum) route infection occurs during passage through the birth canal.
A risk factor for intrauterine infection of the fetus is severe somatic, obstetric, gynaecological, and infectious diseases. At the same time, the risk of infection increases significantly in case of inflammatory diseases of the urogenital tract, adverse pregnancy course (infectious diseases, severe gestosis, threat of termination, pathological condition of the uteroplacental barrier) and abnormal deliveries.

\section{MATERIALS AND METHODS}

Clinical manifestations of TORCH syndrome in the vast majority of cases depend not only on the aetiology but also the timing of pregnancy when the infection occurred. Very often children are born prematurely or with signs of delayed intrauterine development and hepatosplenomegaly. Jaundice, exanthemas, respiratory and various neurological disorders, hemorrhagic syndromes and anaemia are common features. We must emphasize the extremely low reliability of the diagnosis if only clinical features of the disease are analyzed. Laboratory verification of the aetiology of TORCH syndrome is a key part of the diagnosis and determines the possibility to prescribe a specific therapy in time. At the same time, the uniformity of clinical manifestations of intrauterine infection justifies the necessity of urgent laboratory deciphering of the aetiology of the disease. For etiological verification of congenital infections two main groups of methods are used, conventionally designated as "direct" and "indirect".

The "direct" laboratory tests include methods aimed at detecting the pathogen itself (classical microbiological, virological), its DNA or RNA (molecular biological) or antigens (immunochemical). "Indirect" tests are methods that allow the detection of specific antibodies to pathogen antigens in the patient's serum. In recent years, enzyme 
immunoassay has been used most frequently for this purpose.

Polymerase chain reaction (PCR) is the most frequently used of the "direct" methods (specificity and sensitivity of over 90\%), and of the "indirect" methods, immunoassay (specificity and sensitivity of over $75 \%$ ).

Using the polymerase chain reaction, it is reasonable to use modern techniques for the quantitative determination of DNA or RNA of infectious agents, based on amplification with real-time hybridization-fluorescence detection of the analysis products, and parallel testing of several biological media (blood, cerebrospinal fluid, urine, nasopharyngeal swabs, endotracheal aspirates).

Immunoassay should be performed before the administration of plasma and immunoglobulins. The diagnostic value of immunoassay significantly increases if the examination of the newborn is carried out simultaneously with the examination of the mother with the mandatory determination of the avidity index of specific IgG. The use of "paired sera" to determine the increase in antibody concentration over time is possible only if the newborn has not received blood products (plasma, immunoglobulins.)

Criteria for verification of the aetiology of intrauterine infection is the detection of specific IgM and/or genome (DNA, RNA) of the pathogen in the newborn child. An indirect laboratory sign of intrauterine infection of a particular aetiology is the detection of specific IgG with a low avidity index in the absence of specific IgM. The diagnostic value of low-avid specific IgG is significantly increased if highavid specific IgG is detected in the mother during the parallel serological examination.

\section{Herpes}

All of the major types of the herpes family of viruses can cause herpetic infection in the neonate. However, the term "neonatal herpes" is only used to refer to diseases caused by herpes simplex virus types 1 and 2 . The herpes simplex virus type 2 is the most dangerous for the baby.

The likelihood of infecting the child depends on how long the mother has been infected. The "fresher" the infection, the more likely the child will become infected. If the mother has a rash by the time of delivery, it is an indication of a cesarean section.

\section{Clinical manifestations:}

1. Local form (skin-mucosal) - lesions of the skin, mucous membranes, less often encephalitis.

2. Cerebral form - the clinical picture corresponds to the general signs of intrauterine infection, but there are specific signs: lesions of the eyes and mucous membranes; herpetic encephalitis, which is necrotic, resulting in brain destruction can be up to the hemispheres; pronounced thrombocytopenia with the hemorrhagic syndrome.

3. Disseminated neonatal herpes.

\section{Diagnosis:}

In the diagnosis of neonatal herpes, it is important to assess the specific maternal history. When clinically examining children born to mothers with acute or recurrent genital herpes, an inspection of the skin and mucous membranes must be carried out with particular care. If the newborn has seizures of unclear aetiology, a lumbar puncture is indicated (lymphocytosis and high protein concentration are noted in herpetic encephalitis). If the newborn has a clinic of sepsis with no effect from antibiotics - it is 
necessary to examine for herpes. Among laboratory methods of diagnosis, the gold standard is the isolation of the virus from blood, cerebrospinal fluid (CSF), vesicles by culture method. In cutaneous form, the contents of vesicles or scrapings from the skin can be examined by the immunofluorescent method in order to detect the antigen of the virus. In generalized infection and meningoencephalitis, blood and CSF are tested by $P C R$ polymerase chain reaction. IgG antibody levels are not informative because they are maternal antibodies. IgM levels indicate an acute infection in the newborn.

\section{Treatment:}

For all forms of neonatal herpes, systemic antiviral therapy is indicated because the localized form may precede the generalized form. If antiviral drugs are administered early, the outcome is favourable. Regardless of the form of infection, acyclovir is used. Acyclovir (Zovirax, Viroleks) intravenously for 2-3 weeks, plus anti-herpetic immunoglobulin for two weeks. Vidarabine, florenal, bonafton ointment are used to treat ophthalmoscopes.

\section{Congenital cytomegalovirus infection}

Cytomegalovirus is a virus that is transmitted by all secretions (saliva, urine, blood, tears). Clinical manifestations of the primary infection in pregnant women are nonspecific and may resemble those of an acute respiratory infection. There are a number of factors that contribute to the high frequency of intrauterine infection with cytomegaly virus. These include epidemiological features, such as the significant genetic variability of cytomegalovirus strains, the wide distribution of cytomegalovirus infection in the human population (in the vast majority - as a latentpersistent course), the predominance of subclinical forms in both primary and secondary infection, the variety of mechanisms and routes of transmission. The next factor is the immaturity of the immunity of the fetus and the newborn. Finally, adaptive immune changes in a woman's body during pregnancy (decrease in the functional activity of cellular immunity mechanisms), in which the reactivation of latent-persistent cytomegalovirus infection is possible. Infection most often occurs in childbirth or with the mother's milk. During pregnancy, infection occurs only if the mother first becomes infected during pregnancy.

\section{Clinical manifestations:}

Congenital hepatitis with marked jaundice, severe thrombocytopenia with the hemorrhagic syndrome, meningoencephalitis. Specific signs are calcifications in the subependymal regions of the brain and chorioretinitis. The long-term prognosis is determined by the degree of brain damage. If early meningoencephalitis - children are usually disabled, if hepatitis - early development of cirrhosis, if carditis - chronic heart failure.

\section{Diagnosis:}

Children with symptoms of congenital infection and without clinical manifestations of TORCH syndrome should be examined for cytomegalovirus infection if they are born to women at risk. In newborn infants in the early neonatal period, if cytomegalovirus infection is suspected, the pathogen is first identified by any available method. Most often polymerase chain reaction or virus antigen detection is used, less often virological method is used. Any biological fluid (urine, saliva, blood, tears) can serve as a material for a polymerase chain reaction, however, active cytomegalovirus infection is spoken about only when cytomegalovirus genome is detected by a polymerase chain reaction in blood and cerebrospinal fluid. If the virus DNA is found in other media, the period of the disease cannot 
be assessed unambiguously. To clarify the severity of the process serological methods are used - anti-cytomegalovirus antibodies of classes $M$ and $G$ are determined. It is mandatory to study "paired sera", i.e. control of the study of antibody titers after 3-4 weeks. The detection of class IgM antibodies in the umbilical cord blood and in the baby's blood during the first weeks of life is an important diagnostic indicator. Detection of IgG in the child's blood without comparison with maternal titers is not diagnostically significant, since the transplacental transfer of antibodies from the mother is possible.

\section{Treatment:}

Treatment of congenital cytomegalovirus infection consists of etiotropic and syndromic therapy. The etiotropic therapy is indicated in the active period of congenital cytomegalovirus infection. The drug of choice for etiotropic treatment is Cytotec. Children are given anti-cytomegalovirus immunoglobulin (Cytotec) intravenously 2 $\mathrm{ml} / \mathrm{kg} 2$ times daily every 2 days for 3 weeks

If there is life-threatening, ganciclovir is added intravenously for 14-21 days, although virostatics (antiviral drugs) like ganciclovir, foscarnet are used very rarely because of their high toxicity.

\section{Congenital toxoplasmosis}

Toxoplasma oocysts are usually found in the faeces of cats and goats, from where they get into the external environment. In pregnant women, the disease clinic is mononucleosis or flu-like, with high fever or very prolonged subfebrile fever, and enlarged lymph nodes. Often arthralgia or arthritis joins.

Probability of infecting the fetus: Infection usually occurs if the infection is fresh, and depends on the age of the infection. In the first trimester, the probability is $15 \%$, in the second trimester $30 \%$ and in the third trimester $60 \%$.

\section{Clinical manifestations:}

In the fetus and newborn, the infection can be in two forms: eye and brain damage or generalized toxoplasmosis. In addition to the general signs of infectious toxicosis, hepatitis, meningoencephalitis, eye damage (congenital cataract, maybe glaucoma, optic nerve atrophy) join.

\section{Diagnosis:}

Check-up scheme for toxoplasmosis in newborn children: check for antibodies in case of clinical signs of toxoplasmosis. If no antibodies are found, the test is repeated after 2 weeks, if no antibodies are found on the second test, no further control is necessary. If detected, specific therapy is indicated. If IgM antibodies are detected at the initial study, etiotropic therapy is indicated immediately. If only IgG antibodies are detected, the test is repeated after 4 weeks. Therapy is indicated if the antibody titer rises. If the titer drops, the child does not need treatment, but further monitoring is necessary.

\section{Treatment:}

Treatment for toxoplasmosis can be done antenatally - that is, treating the pregnant woman. If the infection in the first half of pregnancy - spiramycin, Claforan, rovamycin are used. If in the second half of pregnancy chloridine + sulfasalazine + folic acid are used. Treatment of children is effective during periods of circulation of extracystic forms of the parasite in the blood, the drugs have no effect on cystic forms. There is no need for complete sanitation since cystic forms (carriage) provide normal non-sterile immunity. Pyrimethamine preparations in combination with sulfonamides are most effective. Combination preparations are 
available: fansidar, metaquelphine and cotrimoxazole in an age-appropriate dosage. Treatment of newborns involves the following scheme: chloridine + sulfadimesin + folic acid. The course of 4- 6 weeks. During the first year, four courses with a break of 1.5 months, and spiramycin for 1.5 months during the break.

\section{Chlamydia}

WHO data show that $35-50 \%$ of newborn infants whose mothers are infected with $C$. trachomatis develop chlamydial ophthalmia (5 times more frequent than gonococcal), and 11$20 \%$ develop pneumonia. Infection usually occurs in childbirth, and the probability of transmission is $40-70 \%$. The disease does not manifest itself immediately, but after 7-14 days.

\section{Clinical manifestations:}

There are three forms of infection in newborns:

- $\quad$ Persistent latent acute (generalized infection - meningoencephalitis, intrauterine pneumonia, gastroenteritis)

The main manifestations of the disease in the newborn are: nasopharyngitis 25\%; conjunctivitis, resistance to the use of conventional drugs, meets only the therapy with tetracycline ointment; pneumonia 10-15\% mild toxaemia, but a pronounced obstructive syndrome, a fit painful cough; high eosinophilia; proctitis, gastroenteritis - $5 \%$; vulvitis, urethritis $15 \%$.

\section{Treatment:}

A newborn baby may be given Erythromycin suppositories for 24 days or oral Erigeron. Azithromycin can also be used.

\section{Mycoplasmosis}

Mycoplasma infection usually occurs during childbirth. The frequency of detection of the pathogen in pregnant women is $20-50 \%$, the risk of infection of the fetus is not known. Treatment of pregnant women with seropositive mycoplasmosis is carried out after the 16th week of pregnancy, which reduces the rate of infant morbidity.

\section{Clinical manifestations:}

In newborns it manifests as pneumonia, which begins imperceptibly with toxaemia, pallor appears, shortness of breath increases, and only then there are physical findings. On the radiograph, a specific sign is the "snowstorm symptom" - bilateral small focal, in some places confluent pneumonia. Lethality is $15 \%$.

\section{Treatment:}

Newborns are prescribed erythromycin or azithromycin and in severe forms levomycetin.

\section{Congenital rubella syndrome}

If the mother becomes infected in the first 12 weeks, it is best to terminate the pregnancy. The mother should be examined before pregnancy and vaccinated if she is seronegative. If the mother is infected with rubella in the 1st trimester, her child has a $25 \%$ chance, and after the 5th month - 1-2\% to be infected.

\section{Clinical manifestations:}

A typical clinical manifestation is the Greg triad: congenital heart defects such as patent ductus arteriosus, interventricular septal defect, interatrial septal defect, pulmonary artery stenosis, myocardial necrosis, eye defects (cataract, microphthalmia, glaucoma), deafness. In 2/3 of children, congenital rubella occurs at the end of the perinatal period.

\section{Treatment:}

There is no specific therapy; treatment is symptomatic. 
Thus, highly sensitive and highly specific methods of laboratory diagnosis are available, enabling rapid and highly reliable verification of the aetiology of the disease, which determines the possibility of early initiation of etiotropic therapy to improve the prognosis of intrauterine infection.

\section{CONCLUSION}

The most comfortable environment for viruses is young tissue, and if infection occurs, the consequences can be most adverse. Many doctors recommend getting tested for these viruses during pregnancy planning, to protect the health of the child. In the case that the pregnancy has already occurred, it is necessary to get tested for them as early as possible. This is done to determine if there was any infection in the body before the pregnancy occurred, or if the infection occurred later. In the first case, there is no danger for the unborn child.

\section{REFERENCES}

1. Degtyarev D.N., Degtyareva M. V., Kovtun I.Y., Shalamova L.V. (1997). Principles of diagnosis of intrauterine infections in newborns and the tactics of management of children at risk. Moscow: Perinatology Today, - Vol. 3, P. 18-24.

2. Petrikovsky B.M., Medvedev M.V., Yudina E.V. (1999). Congenital malformations. Prenatal diagnosis and tactics. M. RAVUZDPG: Real Time. P.325.

3. Okhotnikova I.M, Ageykin V. A., Lozovskaya L. C. (2001). The significance of intrauterine viral infection in the organ pathology of infants. Medical Scientific and Educational-Methodical Journal. № 5 . pp. 81-87.
4. A.Y. Senchuk, Z.M. Dubossarskaya. (2005). Perinatal infections: a practical handbook. MOSCOW: MIA,. P.318

5. Senchuk A. Y., Dubossarskaya Z. M. (2004). Perinatal Infections: a Practical Manual. MOSCOW: MIA,. P. 448.

6. Vlasyuk B. B. (2010). Morphological diagnosis of intrauterine infections. Textbook. St. Petersburg, P. 47. ISBN 5-00-001976-8/.

7. Volodina N.N., Degtyareva D.N. (1999). Diagnosis and treatment of intrauterine infections. Moscow: Methodological guidelines for neonatologists. P.22 